\title{
Prenatally traumatized mice reveal hippocampal methylation and expression changes of the stress- related genes Crhr1 and Fkbp5
}

\author{
Anne-Christine Plank $\mathbb{D}^{1}$, Stefan Frey ${ }^{1}$, Lukas Andreas Basedow $\mathbb{D}^{2}$, Jalal Solati ${ }^{1}$, Fabio Canneva ${ }^{3}$, \\ Stephan von Hörsten $\mathbb{1}^{3}$, Oliver Kratz ${ }^{1}$, Gunther H. Moll ${ }^{1}$ and Yulia Golub ${ }^{1,2}$
}

\begin{abstract}
In our previous study, we found that prenatal trauma exposure leads to an anxiety phenotype in mouse pups, characterized by increased corticosterone levels and increased anxiety-like behavior. In order to understand the mechanisms by which aversive in utero experience leads to these long-lasting behavioral and neuroendocrine changes, we investigated stress reactivity of prenatally traumatized (PT) mice, as well as the expression and methylation levels of several key regulatory genes of the stress axis in the dorsal hippocampus (dHPC) of the PT embryo and adult mice. We detected increased corticotropin-releasing hormone receptor 1 (Crhr 1 ) and decreased FK506 binding protein 5 (Fkbp5) mRNA levels in the left dHPC of adult PT mice. These alterations were accompanied by a decreased methylation status of the Crhr 1 promoter and an increased methylation status of the Fkbp5 promoter, respectively. Interestingly, the changes in Fkbp5 and Crhr1 mRNA levels were not detected in the embryonic dHPC of PT mice. Together, our findings provide evidence that prenatal trauma has a long-term impact on stress axis function and anxiety phenotype associated with altered Crhr1 and Fkbp5 transcripts and promoter methylation.
\end{abstract}

\section{Introduction}

The developmental triad of growth, maturation, and learning is known to be modulated by an individual's early environment ${ }^{1}$. Accordingly, early life experiences, including exposure to the maternal environment, set the growth trajectory of the child and are recognized as a key factor for disease susceptibility later in life ${ }^{2}$. Developmental programming, defined as the persisting effects of in utero events on tissue structure and function, has been shown to impact psychological, behavioral, neuroendocrine, and cardio-metabolic processes in adulthood (see for review $\left.{ }^{3,4}\right)$. From an evolutionary point of view, developmental programming is an adaptation strategy, intended

\footnotetext{
Correspondence: Yulia Golub (yulia.golub@uniklinikum-dresden.de) ${ }^{1}$ Department of Child and Adolescent Mental Health, University Hospital Erlangen, Schwabachanlage 6 and 10, 91054 Erlangen, Germany 2Department of Child and Adolescent Psychiatry, Faculty of Medicine, Technische Universität Dresden, 01307 Dresden, Germany Full list of author information is available at the end of the article
}

for fitting the individual organism into a prospected "future environment" and hence beneficial for survival. However, when there is a mismatch between early and later life environment, these changes - originally intended to be "beneficial" - all together or in parts suddenly become maladaptive ${ }^{5}$. Such specific and often traumaassociated maladaptation increases an individual's disease susceptibility ${ }^{4,5}$. For instance, children of mothers who experienced a traumatic event during pregnancy are at higher risk to develop a psychiatric disorder later in life ${ }^{6-8}$.

In this context, a considerable body of clinical and preclinical research has focused on trauma-related functional changes of the hypothalamic-pituitary-adrenal (HPA) axis ${ }^{9}$. Activation of the HPA axis is driven by hypothalamic secretion of corticotropin-releasing hormone (CRH), triggering pituitary-derived $\mathrm{ACTH}$ release and ultimately adrenal secretion of cortisol (human)/corticosterone (CORT, rodents). CRH is a key factor in the stress response of the brain, and the CRH/CRHR1 (CRH 
receptor 1) system - along with FK506 binding protein 5 (FKBP5) - is known to be critical in mediating susceptibility to anxiety disorders ${ }^{10}$. In rodents, early life stress has been found to induce an increased anxiety-like behavior and cognitive deficits in association with increased central $\mathrm{CRH}$ and CRHR1 expression ${ }^{11}$. CRH secretion and stress signaling are regulated by a negative feedback loop, which is activated via cortisol binding to the glucocorticoid receptor (GR/NR3C1). The sensitivity of the GR, in turn, is modulated by FKBP5, whose dysregulation has previously been linked to PTSD and other anxiety disorders ${ }^{12}$. In contrast to the early assumption that lowered basal cortisol is a hallmark of PTSD, meta-analyses showed that this holds true only for subgroups of patients and depends on the type and the time point of the trauma ${ }^{13,14}$.

Maternal PTSD has been associated with changes in the HPA axis regulation of the offspring, implicating maternally derived glucocorticoid programming in the intergenerational transmission of trauma-related changes ${ }^{6-8}$. Due to its lipophilic nature, maternal cortisol can pass through the placenta and enter the fetal circulation. Thus, stress-induced high maternal cortisol concentrations can impair the fragile balance of the developing HPA axis of the fetus. Such an impact on the programming of the fetal cortisol responsiveness can permanently influence an individual's reactivity to stressful stimuli during its lifetime $^{7}$. The molecular mechanisms by which prenatal stimuli trigger neurodevelopmental alterations in crucial brain regions are likely to involve epigenetic changes, such as modifications in DNA methylation profiles, resulting in altered expression of target genes ${ }^{9}$.

In our previous work, we established a mouse model of prenatal trauma (PT) based on the application of an electric foot shock to C57BL/6N female mice on the gestational day (GD) 12 during their pregnancy ${ }^{15-17}$. We found high anxiety levels and poor maternal care along with reduced serum prolactin and increased CORT levels in dams following maternal trauma (MT). Moreover, prenatally traumatized pups (PT pups) were born smaller and stayed smaller throughout their life. Additionally, when raised by a traumatized mother, PT pups had higher serum CORT levels and displayed increased anxiety-like behavior ${ }^{17}$.

The present work was conducted to better understand the long-term effects of prenatal trauma and/or being raised by a traumatized mother on the regulation of the stress system. We investigated epigenetic changes in several key regulatory genes of the stress axis in the hippocampus of PT mice, including Crhrl, Fkbp5, $N r 3 c 1$, and Nr3c2 (encoding the mineralocorticoid receptor). In order to distinguish prenatal vs. postnatal effects, we examined samples collected from experimental animals sacrificed at GD 18 and at postnatal day (PND) 150. We focused on changes in the hippocampus, since it is crucially involved in fear memory processes and the regulation of the CRH system ${ }^{18,19}$.

We hypothesized that: (1) PT mice would display increased basal and stress-induced CORT levels; (2) changes in the CORT levels would go along with changes in the expression and methylation levels of key HPA axis regulatory genes; and (3) changes in the expression levels of the HPA axis regulatory genes following PT could be detected in embryos following in utero trauma.

\section{Material and methods \\ Animals}

Adult male and female $\mathrm{C} 57 \mathrm{Bl} / 6 \mathrm{NCrl}$ mice were purchased from Charles River Germany GmbH (Sulzfeld, Germany) at the age of 8 weeks. Animals were housed under standard conditions with a 12:12-h light/dark cycle (lights on at 05:00 a.m.), controlled temperature (23 \pm $\left.1{ }^{\circ} \mathrm{C}\right)$ and with food and water ad libitum.

Breeding (1 female and 1 male per cage) was initiated at the age of 10 weeks after 2 weeks of acclimatization. GD 0 was counted on the 2nd day of pairing with the male, and putatively pregnant females were single housed and monitored daily to ascertain their condition. Pups were reared by their natural mothers, weaned on PND 25, and housed in groups of four with no more than two animals of the same litter. Experiments were performed with male offspring, and a maximum of two male mice per litter were included in a single experiment to avoid "litter effects". Animals were randomly assigned to their respective group by a chance procedure. The experimenter was not blinded to the group status. Group sample size determination was based on respective power analyses performed for our previously published studies ${ }^{15-17}$. All experimental procedures were approved by the district governments of Middle Franconia (Regierung von Mittelfranken, Ansbach, Az 54-2532.1-32/10) and Lower Franconia (Regierung von Unterfranken, Würzburg, Az 55.2-2532-2-441), Bavaria, Germany, and performed in strict compliance with the European Union Directive for the care and use of laboratory animals (ARRIVE guidelines ${ }^{20}$ ).

\section{Experimental procedures \\ Trauma application}

MT was induced by contextual fear conditioning (Multi-Conditioning System, TSE Systems, Bad Homburg, Germany) at GD 12 (corresponding to the second trimester of gestation in humans), as previously described ${ }^{17}$. Animals were placed into the shock chamber for application of an inescapable foot shock: following $198 \mathrm{~s}$ of habituation, a single electric foot shock $(2 \mathrm{~s}, 1.5 \mathrm{~mA})$ was delivered via the metal grid. Animals remained in the chamber for $60 \mathrm{~s}$ before being returned to their home cages. The control group (no MT) was exposed to the 
shock environment without an electrical shock delivery. After each trial, the fear conditioning boxes were cleaned thoroughly with $70 \% \mathrm{EtOH}$. The test was performed between 6 and 8 p.m., i.e., during the dark phase.

\section{Elevated plus maze (EPM)}

In order to measure stress reactivity, mice were exposed to a classical EPM paradigm, performed as previously described $^{17}$. The EPM was composed of two open arms $(30 \times 5 \mathrm{~cm})$, and two enclosed arms $(30 \times 5 \times$ $15 \mathrm{~cm}$ ) with an open roof and elevated to the height of $50 \mathrm{~cm}$ above the floor. Mice were placed individually in the center of the maze, facing one of the open arms and their behavioral performance was recorded for $5 \mathrm{~min}$ by a camera positioned $1.5 \mathrm{~m}$ above the center of the maze. The test was performed between 11 a.m. and 2 p.m. during the light phase.

\section{Experiments}

Experiment 1: the impact of PT on basal and stress-induced CORT levels and on hippocampal expression and methylation levels of HPA axis key genes in adult mice

Pregnant dams were subjected to trauma (MT; $n=14$ ) or alternatively exposed to the shock chamber without application of the shock (no MT; $n=15$ ), as described above (see Fig. 1A). Prenatally traumatized and control mice were subjected to further experimental procedures at the age of 21.5 weeks (PND 150), dams were tested 1 week after weaning (age: 18 weeks) (Fig. 1A):

- Dams were exposed to the mild stress of one EPM session for CORT measurements. Blood samples from each animal were taken at two different time points: basal (7 days before EPM session, immediately after separation from their pups) and post stress (60 min after behavioral testing). Blood was obtained from each animal (no MT: $n=13$; MT: $n=12$ ) via facial vein puncture (basal) and from the retro-orbital vein plexus (post stress) using hematocrit tubes under isoflurane narcosis. All sampling took place between 11 a.m. and 1 p.m. Serum preparation and CORT measurement were performed as described below.

- Similarly, basal (7 days before EPM session) and stress-induced (60 min after EPM session) serum CORT levels were measured in one group of PT male offspring $(n=10)$ and no PT control animals $(n=10)$. Blood was obtained from each animal via facial vein puncture (basal) and from the retroorbital vein plexus (post stress) as described above.

- For gene expression and methylation analyses, tissue samples of PT and no PT mice (gene expression analyses: $n=7$ per group; methylation analyses: $n=10$ per group) were collected within $12 \mathrm{~min}$ after disturbing the cage. Animals were sacrificed via decapitation under short-term sedation with isoflurane (maximum exposure time: $10 \mathrm{~s}$ ) and the brain was immediately isolated and shock frozen in liquid nitrogen. Frozen brains were coronally fixed and precut in a cryostat (CM3050S, Leica Biosystems, Wetzlar, Germany), followed by punching out a sample of the dorsal hippocampus (dHPC), which was defined based on a stereotaxic atlas $(1.2 \mathrm{~mm}$ for $\mathrm{dHPC}$, starting at $1.6 \mathrm{~mm}$ posterior to bregma). The dHPC samples were immediately frozen in liquid nitrogen and stored at $-80^{\circ} \mathrm{C}$ until further processing. The subsequent experimental procedures are described in detail below.

\section{Experiment 2: the impact of prenatal trauma on embryonic expression levels of HPA axis key genes in the AHPC}

In order to investigate whether gene expression changes in the dHPC occurred before birth, pregnant female mice $(n=7)$ were subjected to MT or exposed to the shock chamber without an application of the shock (no MT, $n=7$ ) as described above (see Fig. 1B). On GD 18, 6 days after trauma exposure, pregnant mice were sacrificed and the fetuses isolated. Tissue sampling took place between 11 a.m. and 1 p.m. to ensure constant conditions and handling. Freshly isolated fetal brains were stored in RNAlater (Thermo Fisher, Waltham, MA, USA) for $24 \mathrm{~h}$ at $4{ }^{\circ} \mathrm{C}$ before dHPC isolation to prevent RNA degradation during the isolation process. Sampling of fetal dHPC tissue on GD 18 (no PT: $n=8$; PT: $n=8$ ) was performed as described in detail by Seibenhener and Wooten ${ }^{21}$. Hippocampal specimens were shock frozen in liquid nitrogen and stored at $-80^{\circ} \mathrm{C}$ until further processing.

\section{Corticosterone measurements}

Blood was incubated for $45 \mathrm{~min}$ at room temperature to allow coagulation, and serum was subsequently separated at $2000 \times g$ for $10 \mathrm{~min}$, aliquoted and stored at $-80^{\circ} \mathrm{C}$ until the measurements were performed. CORT concentrations were determined using commercial ELISA kits (RE52211, IBL international GmbH, Hamburg, Germany) following the manufacturer's instructions. All samples were assayed in duplicate using a microplate reader with corresponding software (Benchmark Plus ${ }^{\mathrm{TM}}$ microplate spectrophotometer, Bio-Rad Laboratories $\mathrm{GmbH}$, Hercules, CA, USA) and quantified against a standard curve.

\section{RNA isolation and CDNA synthesis}

RNA from dHPC specimens was extracted using the RNeasy Mini Kit (Qiagen, Hilden, Germany) according to the manufacturer's instructions. Subsequently, RNA quality was determined by 260/230 and 260/280 ratios (Eppendorf 

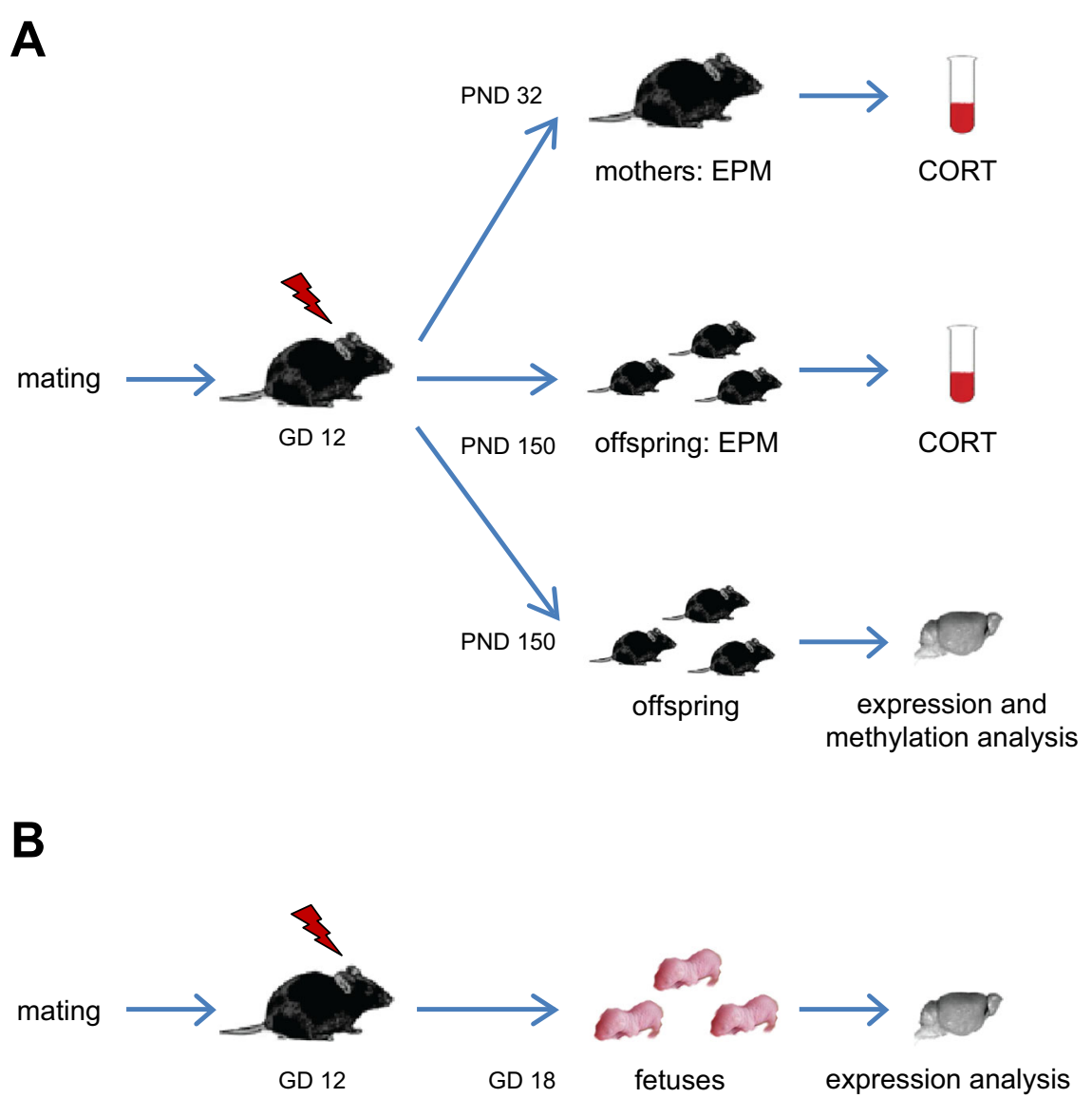

Fig. 1 Experimental scheme. Schematic overview of experiment 1 (A) and experiment 2 (B). Lightning symbol = traumatic experience (electric foot shock) of the MT group; GD gestational day, PND postnatal day, EPM elevated plus maze.

Biophotometer, Eppendorf, Hamburg, Germany), as well as by gel electrophoresis. RNA samples, which fulfilled the MIQE guidelines ${ }^{22}$, were used for cDNA synthesis according to manufacturer's protocol (Thermo Fisher, Waltham, MA, USA).

\section{qPCR}

$10 \mathrm{ng}$ of cDNA was used per sample and gene for expression analyses of $\mathrm{Crhr} 1, \mathrm{Fkbp5}, \mathrm{Nr} 3 \mathrm{c} 1$, and $\mathrm{Nr} 3 \mathrm{c} 2$ by qPCR. Each sample was measured in triplicate using $\mathrm{iQ}^{\mathrm{TM}}$ $\mathrm{SYBR}^{\circledR}$ Green Supermix (Bio-Rad Laboratories $\mathrm{GmbH}$, Hercules, CA, USA) on a CFX95 ${ }^{\mathrm{TM}}$ real-time cycler (BioRad Laboratories GmbH, Hercules, CA, USA) running customized software. Cqs (Cycle at which the signal intersects the threshold between real signal and background) were determined by regression analysis and $\Delta \mathrm{Cq}$ values calculated on account of two reference genes (TATA box binding protein $(T b p)$ and beta-Actin $(A c t b)$ ). According to quality standards, triplicates with a standard deviation $\geq 0.2$ cycles were excluded. Outliers were identified using median $\pm 3 *$ MAD for each sample. Expression folds were calculated using the $2^{-\Delta \mathrm{Cq}}$ method and considering the respective primer efficiency. The following primer pairs were used:

Crhr1: fw: 5'-GCCCCATGATCCTGGTCCTGC-3', rev: 5'-CCATCGCCGCCACCTCTTCC-3';

Fkbp5: fw: 5'-GTGGGTTCTACATCGGCACT-3', rev: 5'-GAGTCTGCGAAAGGACTTGG-3';

Nr3c1: fw: 5'-AACTGGAATAGGTGCCA AGG-3', rev: 5'-GAGGAGAACTCACATCTGGT-3';

Nr3c2: fw: 5'-CATGGAGATTGTCAACGTCA-3'; rev: 5'-CTC GGCATCTCTCACAGAAT-3';

Tbp: fw: 5'-CCTATCACTCCTGCCACACC-3'; rev: 5'ATGACTGCAGCAAATCGC TTG-3';

Actb: fw: 5'-GGCACCACACCTTCTACAATG-3'; rev: 5'-GGGGTGTTGAAGGTCTCAAAC-3'.

\section{Methylation analysis}

Genomic DNA was isolated from dHPC samples using a commercial kit (QIAamp DNA Micro Kit, Qiagen, Hilden, Germany) following the manufacturer's instructions and sent to Varionostic GmbH (Ulm, Germany) for EpiTYPER $^{\circledR}$ (Agena Bioscience, CA, USA) methylation analyses. Genomic DNA was treated with bisulfite to 
A

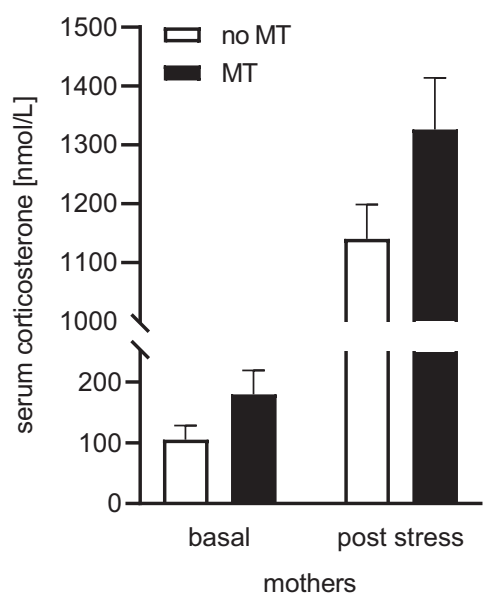

B

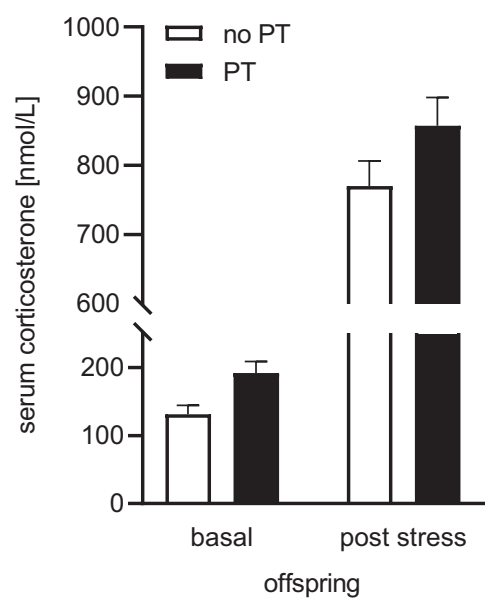

Fig. 2 Serum CORT levels of MT and PT mice before and after stress exposure. A Basal CORT measurement of dams was performed 4 weeks after birth and 7 days before exposure to mild stress. MT dams showed increased basal and post stress CORT levels as compared to control animals. B In PT adult male mice, basal and post stress serum CORT levels were also higher than in no PT mice. See "Results" for detailed statistical analysis.

convert non-methylated cytosine to uracil, resulting in methylation-dependent sequence variations of $\mathrm{C}$ to $\mathrm{T}$. These $\mathrm{C} / \mathrm{T}$ variations appear as G/A changes on the reverse strand, which result in a mass difference of $16 \mathrm{Da}$ per CpG site and can subsequently be detected by the MassARRAY system. The treated DNA was amplified by PCR and treated with shrimp alkaline phosphatase to neutralize unincorporated dNTPs. Subsequently, in vitro RNA transcription was performed, followed by basespecific cleavage at uracil residues using RNase A. The resulting fragments differ in mass and size, depending on the sequence changes due to bisulfite treatment, and generate characteristic signal patterns, which were identified by MALDI-TOF mass spectrometry.

\section{Data analysis and statistics}

Data were analyzed using SPSS 21.0 (IBM, Armonk, NY, USA) and GraphPad Prism 8.0 (GraphPad Software Inc., San Diego, CA, USA). For CORT level analyses, normal distribution of data was confirmed by a Shapiro-Wilk test and a repeated measures two-way ANOVA (factors "MT"/"PT" and "pre/post stress") was applied. For methylation analyses, two-tailed independent samples $t$-tests or $u$-tests, depending on a preceding test of normality (Shapiro-Wilk test), were used and $p$ values were adjusted by false discovery rate procedure. Gene expression data were confirmed to be normally distributed (Shapiro-Wilk test) and analyzed via two-tailed independent samples $t$-test (fetal gene expression) or two-way ANOVA (factors "PT" and "hemisphere") and post hoc Fisher's LSD test. Similarity of variances between compared groups was confirmed by respective $F$ tests. Values larger or smaller than the group mean \pm 2 standard deviations were excluded from statistical analysis. Data are presented as mean values \pm SEM. Statistical significance was accepted at $p<0.05$.

\section{Results}

MT/PT affects basal and stress-induced CORT secretion

CORT measurements in dams 7 days before and $60 \mathrm{~min}$ after mild stress exposure revealed a significant effect of MT $\left(F_{1,23}=5.389, p=0.03\right)$ and of stress exposure $\left(F_{1,23}=\right.$ 363.5, $p<0.0001)$, with increased CORT levels after mild stress exposure and elevated CORT levels in MT as compared to no MT mice (Fig. 2A). However, no interaction of both factors was revealed $\left(F_{1,23}=0.948, p=0.34\right.$ [n.s.])

In their offspring, stress exposure also caused a significant increase in CORT levels, both in PT and no PT mice $\left(F_{1,18}=745.6, p<0.0001\right)$ (Fig. $\left.2 \mathrm{~B}\right)$. Additionally, PT displayed higher CORT levels than no PT mice $\left(F_{1,18}=\right.$ 4.767, $p=0.043$ ) (Fig. 2B). Again, no interaction effect was detected $\left(F_{1,18}=0.31, p=0.584\right.$ [n.s.]).

\section{PT and/or being raised by a traumatized mother is} associated with long-term changes in hippocampal gene expression

In order to study the mechanism of HPA axis changes following prenatal trauma exposure and being raised by a traumatized mother, we measured the expression and methylation changes of the HPA axis key genes Crhrl, $N r 3 c 1, N r 3 c 2$, and $F k b p 5$ in the left and right dHPC (the punched areas are outlined in Fig. 3A) of PT and no PT mice on PND 150. Analysis of Crhr1 expression identified a significant effect of PT $\left(F_{1,24}=8.422, p=0.008\right)$ and an 


\section{A}

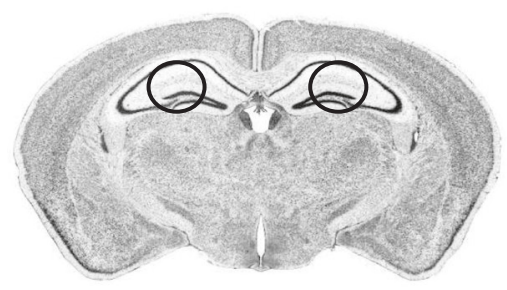

B

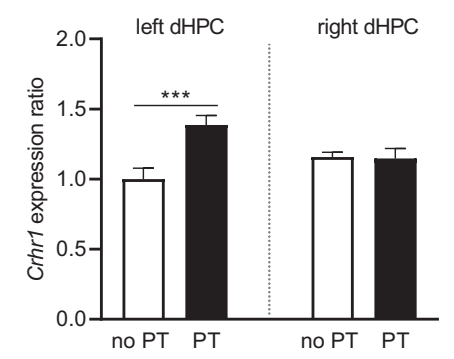

C

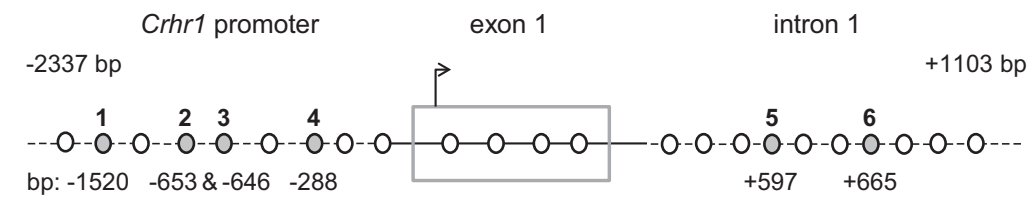

D

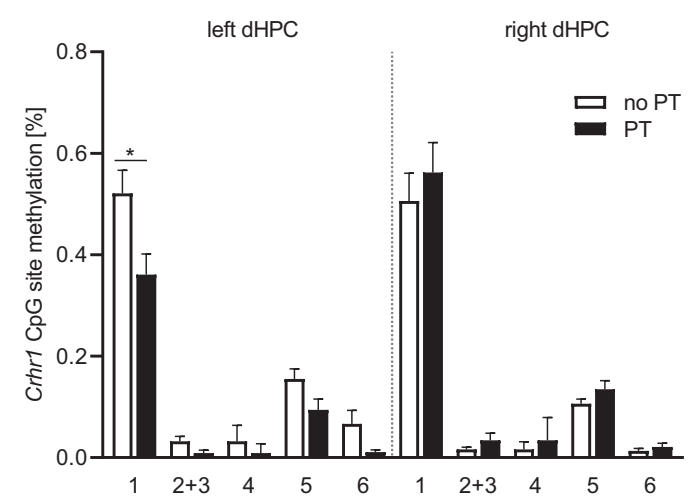

Fig. 3 Expression and methylation analysis of $\mathrm{Crhr} 1$ in the dHPC of PT adult male mice compared to a no PT control group. A

Representative microphotograph with labels of the dissected dHPC region. B Results of the expression analysis of Crhrl in the left and right dHPC of PT adult male mice compared to a control group. The dotted gray line separates the results from left and right dHPC. Crhr1 expression was significantly increased in the left dHPC of PT offspring ( ${ }^{* *} p<0.001$, post hoc Fisher's LSD test), but not changed in the right dHPC as compared to no PT mice. C Schematic representation of the Crhrr region of interest and localization of CpG sites (white and gray circles) investigated by methylation analysis. The promoter, the first exon and intron of Crhrl were analyzed for methylation changes. The gray circles indicate the position of differentially methylated $\mathrm{CpG}$ sites as identified by bisulfite conversion and mass spectroscopy. The shown positions of the respective CpG sites are based on NC_000077.6 (Mus musculus strain C57BL/6J chromosome 11, GRCm38.p2). D Differentially methylated Crhr1 CpG sites in the left and right dHPC of PT and no PT mice. The dotted gray line separates the results from left and right dHPC. CpG site $2+3$ are in juxtaposition to each other and thus have been analyzed together due to technical limitations. * $p<0.05$, independent samples $t / u$-test. See "Results" for detailed statistical analysis.

interaction of the factors $\mathrm{PT}$ and hemisphere $\left(F_{1,24}=\right.$ 9.348, $p=0.005)$, with a significant increase of Crhr1 mRNA levels in the left $\mathrm{dHPC}$ of PT offspring as compared to no PT mice $(p<0.001$, Fisher's LSD test) and compared to the right dHPC ( $p=0.016$, Fisher's LSD test) (Fig. 3B). No trauma-related differences in Crhr1 mRNA levels were detected between no PT and PT mice regarding the right $\mathrm{dHPC}(p=0.914$ [n.s.], Fisher's LSD test), and Crhr1 expression in the dHPC of no PT offspring did not differ significantly between hemispheres either ( $p=0.098$ [n.s.], Fisher's LSD test).

Additionally, two-way ANOVA revealed a significant effect of PT $\left(F_{1,22}=9.121, p=0.006\right)$ and an interaction of the factors PT and hemisphere $\left(F_{1,22}=7.229\right.$, $p=0.013$ ) for $F k b p 5$ expression, which was significantly decreased in the left dHPC of PT mice (left dHPC, no PT 


\section{A}

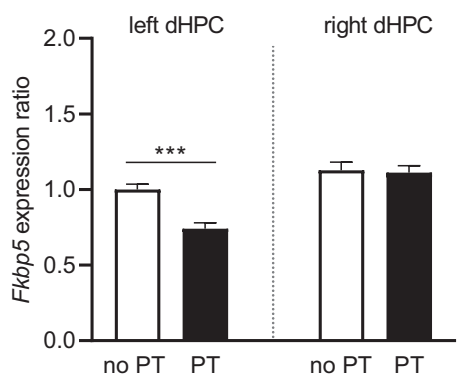

B

Fkbp5 promoter

exon 1

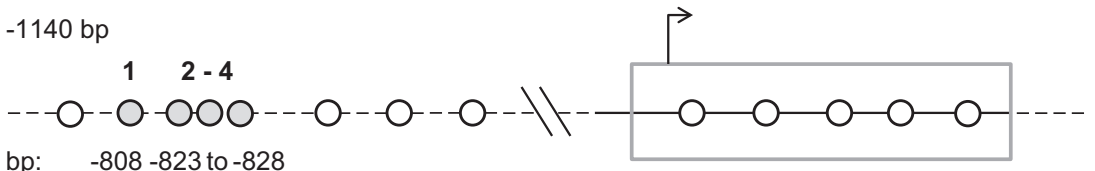

bp: $\quad-808-823$ to -828

C

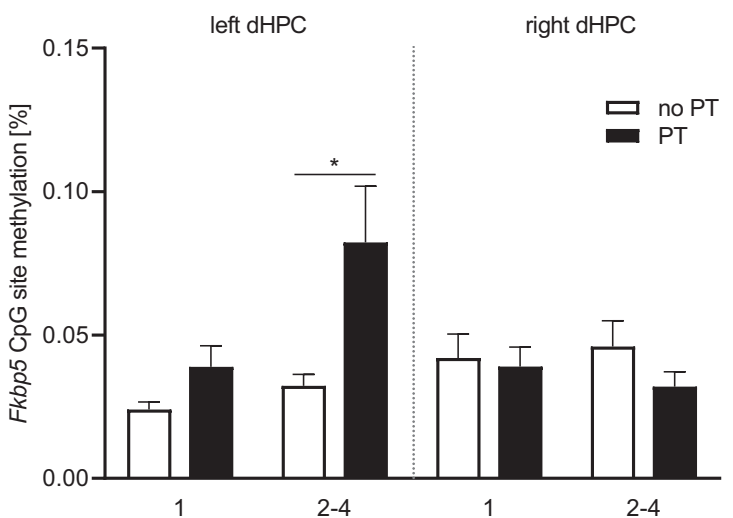

Fig. 4 Expression and methylation analysis of $F k b p 5$ in in the dHPC of PT adult male mice compared to a no PT control group. A Expression analysis of Fkbp5 in the left and right dHPC (the dotted gray line separates the results from left and right dHPC). Fkbp5 expression was significantly decreased in the left dHPC of PT offspring $\left(^{* *} p<0.001\right.$, post hoc Fisher's LSD test), but not changed in the right dHPC as compared to no PT mice. B The promoter and the first exon of Fkbp5 were analyzed for methylation changes. The gray circles indicate the differentially methylated CpG sites. The positions of the respective CpG sites are based on NC_000083.6 (Mus musculus strain C57BL/6J chromosome 17, GRCm38.p4). C Differentially methylated Fkbp5 $\mathrm{CpG}$ sites in the left and right dHPC of PT and no PT mice. The dotted gray line separates the results from left and right dHPC. CpG sites 2-4 are in juxtaposition to each other and thus have been analyzed together due to technical limitations. ${ }^{*} p<0.05$, independent samples $t / u$ test. See "Results" for detailed statistical analysis.

vs. PT: $p<0.001$; PT, left vs. right dHPC: $p<0.0001$, Fisher's LSD test) (Fig. 4A). Comparing the right dHPC of PT and no PT mice, as well as both hemispheres of the latter, $F k b p 5$ expression was not significantly altered (right dHPC, no PT vs. PT: $p=0.817$ [n.s.]; no PT, left vs. right dHPC: $p=0.061$ [n.s.], Fisher's LSD test) (Fig. 4A).

Regarding the expression of $\mathrm{Nr} 3 c 1$ and $N r 3 c 2$, no differences were detected in the right and left dHPC (data not shown).
PT and/or being raised by a traumatized mother is associated with long-term changes in hippocampal gene methylation

Methylation analysis of the promoter region of Crhr1 (Fig. 3C) in the left dHPC revealed a significant hypomethylation in CpG site \#1 at -1520 bp $(t=2.628, \mathrm{df}=$ $18, p=0.02)$ and in two CpG sites with a trend toward significance $(t=2.73, \mathrm{df}=16, p=0.06 ; t=2.09, \mathrm{df}=13$, $p=0.06$ ) in PT mice (Fig. 3D). Moreover, we identified 
A

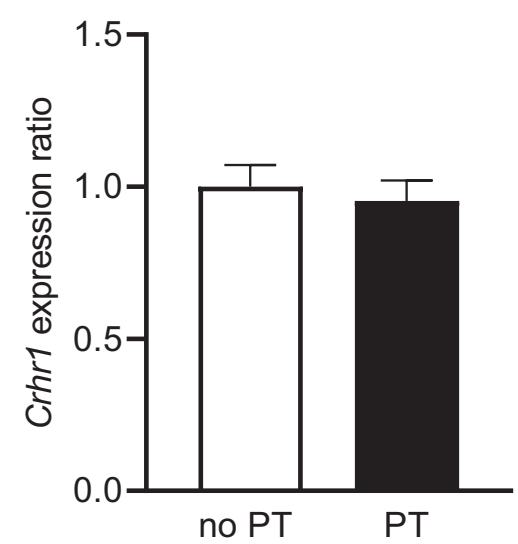

B

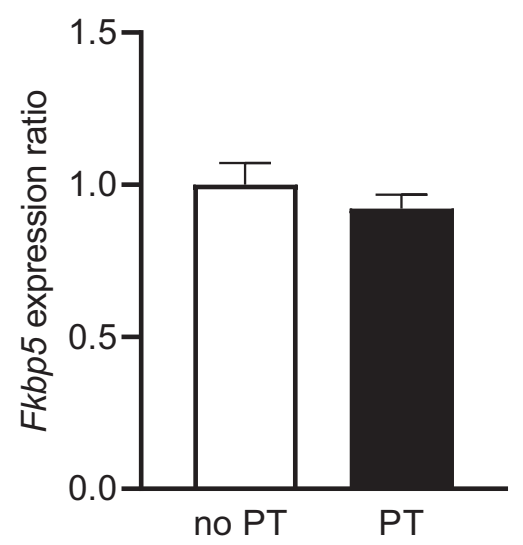

Fig. 5 Expression analysis of Crhr1 and Fkbp5 in the dHPC of PT fetuses compared to a no PT control group. Expression analysis of A Crhr 1 and $\mathbf{B}$ Fkbp5 in the dHPC of male fetuses on GD 18 revealed no PT-related alterations. See "Results" for detailed statistical analysis.

two additional hypomethylated $\mathrm{CpG}$ sites in the first intron of Crhr1 (Fig. 3C); however, differences did not reach statistical significance $(t=1.989, \mathrm{df}=13, p=0.06$; $t=1.988, \mathrm{df}=18, p=0.06$ ) (Fig. 3D). No methylation differences between PT and the control group were identified in the right dHPC (Fig. 3D). The analysis of the methylation status of the Fkbp5 promoter region (Fig. 4B) revealed a significant hypermethylation of CpG sites \#2-4 $(t=2.495, \mathrm{df}=16, p=0.02)$ and a trend to hypermethylation at CpG site \#1 ( $t=1.985, \mathrm{df}=17, p=0.06)$ in the left dHPC of adult PT mice (Fig. 4C). No such differences were observed at $\mathrm{CpG}$ sites in the right $\mathrm{dHPC}$ of the same animals (Fig. 4C). Also, no PT-related changes in the methylation patterns were detected for the investigated regions of $N r 3 c 1$ and $N r 3 c 2$ in the dHPC (data not shown).

\section{No expression changes of HPA axis-related genes in the fetal dHPC following PT}

To examine whether or not the observed expression changes in the adult $\mathrm{AHPC}$ already occur in utero, we performed qPCR analysis in the $\mathrm{dHPC}$ of PT and control fetuses on GD 18, i.e., 6 days after MT procedures.

However, the changes in Crhr1 and Fkbp5 mRNA levels observed in the $\mathrm{AHPC}$ of adult PT and no PT mice were not detected in the dHPC of PT fetuses on GD 18 (Crhr1: $t=0.478, \quad \mathrm{df}=13, \quad p=0.64 \quad$ [n.s.]; Fkbp5: $t=0.950$, $\mathrm{df}=13, p=0.359$ [n.s.]) (Fig. 5A, B). We also did not find PT-related differences with regard to the hippocampal expression of $N r 3 c 1$ and $N r 3 c 2$ (data not shown).

\section{Discussion}

In the present work, we used our previously established mouse model of prenatal trauma ${ }^{17}$ in order to investigate the impact of PT on the regulation of the stress system. Previously, we demonstrated that PT pups are born smaller and remain smaller throughout their life ${ }^{17}$ and that, when raised by traumatized mothers, PT pups display higher circulating CORT levels and increased anxiety-like behavior. Additionally, we observed high anxiety levels and poor maternal care along with reduced serum prolactin and elevated serum CORT concentrations in dams following $\mathrm{MT}^{17}$.

In the present study, the expression levels and methylation profiles of several key regulatory genes of the stress axis were assessed in the $\mathrm{dHPC}$ of PT mice in order to understand the mechanisms by which prenatal traumatic experience leads to increased CORT and anxiety levels. We confirmed our previous findings showing that maternal traumatic experience causes HPA axis dysregulation, manifesting in increased basal and stress-induced CORT levels, both in traumatized mothers and their offspring. We failed to detect a significant interaction of stress and trauma with regard to CORT levels, indicating a trauma-induced increase in CORT levels but not in CORT reactivity.

We further detected increased basal Crhr1 mRNA levels in the left dHPC of PT mice. Notably, these changes in gene expression were accompanied by a correspondingly altered methylation pattern, namely, hypomethylated CpG sites in the Crhr1 promoter region. Crhrl is widely expressed in the mammalian brain and involved in the activation of the HPA axis ${ }^{23}$. Interestingly, regarding the increased anxiety-like behavior of PT mice, it has been suggested that higher levels of CRHR1 induce an anxietylike phenotype in rodents ${ }^{11}$. Moreover, Labermeier et al. ${ }^{24}$ reported on an impact of increased Crhrl expression on stress vulnerability in male mice ${ }^{24}$. Our present results are in agreement with these findings, further corroborating a potential role of Crhr1 in the higher risk of developing anxiety disorders following prenatal trauma and/or being raised by a traumatized mother. 
We were also able to detect a decrease in the expression levels of $F k b p 5$ in the left dHPC, along with a significant hypermethylation of three CpG sites in the Fkbp5 promoter region in the left $\mathrm{dHPC}$ of adult PT mice. Though Fkbp5 regulation can clearly be seen from a developmental perspective as having a life-long, potentially dynamic modulation ${ }^{25}$, our findings were unexpected in terms of their direction since previous studies have shown that early life trauma and early life stress exposure in rodents induce $F k b p 5$ demethylation and upregulation of Fkbp5 expression in the amygdala, leading to GRresistance and a prolonged stress response ${ }^{26}$.

However, Fkbp5 methylation and expression might be subject to specific and differential regulation in individual limbic brain regions. For instance, Szymanska et al. ${ }^{27}$ found that prenatal trauma resulted in HPA axis hyperactivity in prenatally stressed rats, along with an increased density of GR and nonsignificantly lowered levels of FKBP51 in the hippocampus, whereas in the frontal cortex, FKBP51 levels were significantly decreased and those of GR unchanged ${ }^{27}$. These results were considered surprising, since the hippocampus predominantly exerts an inhibitory effect on HPA axis activity ${ }^{28}$, and the lack of such effect - resulting in HPA axis hyperactivity, as observed in PT rats - should rather be associated with reduced GR in prenatally stressed animals ${ }^{27}$. However, our findings in PT mice point in a similar direction, suggesting a PT-related hippocampal dysregulation. Interestingly, Wei et al. ${ }^{29,30}$ demonstrated that overexpression of GR in the forebrain of mice resulted in normal basal CORT levels, but in an increased anxietylike behavior and a deficit in negative feedback with elevated circulating CORT after chronic stress exposure ${ }^{29,30}$. Considering that stress processing involves complex, integrated circuit regulation ${ }^{28}$, we hypothesize that PT might have a different impact on $F k b p 5$ expression in the dHPC than in the amygdala and likely involves distinct epigenetic changes that need to be investigated by longitudinal studies, dissecting the effects of trauma exposure, adaptation processes, and subsequent development of the disease.

Surprisingly, we found that these methylation and expression differences were present only in the left, but not the right dHPC. However, Shipton et al. ${ }^{31}$ observed an effect of the left HPC on associative spatial long-term memory, which did not occur after manipulating the right $\mathrm{HPC}^{31}$. These findings support the idea of a lateralized long-term memory processing in mice, which, in turn, could explain why methylation differences in Crhr1 and Fkbp5 are only detectable in the left dHPC.

The range of these changes in DNA methylation lay between 2 and $10 \%$. This is consistent with findings of others, supporting a model of even small changes $(\leq 10 \%)$ in DNA methylation contributing to complex disease phenotypes $^{32-34}$. For instance, Sudermann et al. ${ }^{35}$ identified a significant impact of early life stress on $N r 3 c 1$ gene expression and HPA axis responsiveness in rats, which was based on small methylation changes $(2-4 \%)$ in the promoter region of $\mathrm{Nr}_{3} \mathrm{cl}^{35}$. In contrast to these findings and those of Müller et al. ${ }^{36}$, who reported on Crhr1dependent $\mathrm{Nr} 3 \mathrm{c} 2$ expression in the $\mathrm{dHPC}$ of mice ${ }^{36}$, we could not detect a significant effect of PT on the expression and methylation of $\mathrm{Nr} 3 \mathrm{cl}$ or $\mathrm{Nr} 3 \mathrm{c} 2$, although Crhr1 expression was altered. However, this might be explained by the different experimental setting of our study, since we analyzed basal gene expression, whereas Müller et al. ${ }^{36}$ focused on stress-induced changes.

Exposure of the pups to increased maternal CORT concentrations through the blood has been discussed as a possible mechanism for effects of PT. Here, we investigated whether the observed alterations in gene expression can already be found prenatally (i.e., shortly after trauma and independently of the postnatal environment). Since the changes in Fkbp5 and Crhr1 mRNA levels in the dHPC were not detected at GD 18, they are likely to occur after birth. However, we are aware that the validity of this conclusion is limited, as due to technical reasons, fetal gene expression in the dHPC was analyzed without distinction between hemispheres. We aim to clarify this issue in future studies and to investigate whether prenatally unaltered expression of the respective genes is due to a delayed manifestation or to being raised by a traumatized mother. Moreover, future work should include the examination of protein levels to complete and support our results obtained by gene expression and methylation analyses, which we focused on in the present work due to a limited number of $\mathrm{PT} /$ no PT offspring and thus availability of $\mathrm{dHPC}$ samples.

In summary, prenatal trauma is known to have an impact on HPA axis functioning, and accordingly, our study revealed increased Crhr1 and decreased Fkbp5 expression in the left dHPC of PT mice. These differences in gene expression were accompanied by corresponding hypo- and hypermethylation of $\mathrm{CpG}$ sites in the promoter regions of Crhr1 and Fkbp5, respectively. Moreover, we detected an increased basal and post stress CORT secretion in these animals. Our findings provide evidence that prenatal trauma and/or being raised by a traumatized mother has a long-term epigenetic impact on stress axis function and anxiety phenotype of the offspring.

\footnotetext{
Acknowledgements

The authors thank Jörg Distler and Ruth Steigleder for their contribution to the project. We acknowledge support by the Friedrich-Alexander-Universität Erlangen-Nürnberg (FAU) within the scope of the funding programs "Erlanger Leistungsbezogene Anschubfinanzierung und Nachwuchsförderung" and "Nachwuchsförderung" to Y.G. and S.F.
}

\section{Author details}

${ }^{1}$ Department of Child and Adolescent Mental Health, University Hospital Erlangen, Schwabachanlage 6 and 10, 91054 Erlangen, Germany. ${ }^{2}$ Department of Child and Adolescent Psychiatry, Faculty of Medicine, Technische Universität Dresden, 01307 Dresden, Germany. ${ }^{3}$ Department Experimental Therapy, University Hospital Erlangen and Preclinical Experimental Animal Center, 
Friedrich-Alexander-University Erlangen-Nürnberg, Palmsanlage 5, 91054 Erlangen, Germany

\section{Funding}

Open Access funding enabled and organized by Projekt DEAL.

\section{Conflict of interest}

The authors declare no competing interests.

\section{Publisher's note}

Springer Nature remains neutral with regard to jurisdictional claims in published maps and institutional affiliations.

Received: 10 June 2020 Revised: 11 January 2021 Accepted: 1 February 2021

Published online: 23 March 2021

\section{References}

1. Hofer, M. A. Early relationships as regulators of infant physiology and behavior. Acta Paediatr. Suppl. 397, 9-18 (1994).

2. Szyf, M. \& Bick, J. DNA methylation: a mechanism for embedding early life experiences in the genome. Child Dev. 84, 49-57 (2013).

3. Nemoda, Z. \& Szyf, M. Epigenetic alterations and prenatal maternal depression. Birth Defects Res. 109, 888-897 (2017).

4. Van den Bergh, B. R. H. et al. Prenatal developmental origins of behavior and mental health: the influence of maternal stress in pregnancy. Neurosci Biobehav Rev. 117, 26-64 (2020).

5. Gluckman, P. D., Cutfield, W., Hofman, P. \& Hanson, M. A. The fetal, neonatal, and infant environments-the long-term consequences for disease risk. Early Hum. Dev. 81, 51-59 (2005).

6. O'Donnell, K, O'Connor, T. G. \& Glover, V. Prenatal stress and neurodevelopment of the child: focus on the HPA axis and role of the placenta. Dev. Neurosci. 31, 285-292 (2009).

7. Yehuda, R. \& Bierer, L. M. Transgenerational transmission of cortisol and PTSD risk. Prog. Brain Res. 167, 121-135 (2008).

8. Lehrner, A. et al. Maternal PTSD associates with greater glucocorticoid sensitivity in offspring of Holocaust survivors. Psychoneuroendocrinology 40, 213-220 (2014).

9. Klengel, T. \& Binder, E. B. Epigenetics of stress-related psychiatric disorders and gene $x$ environment interactions. Neuron 86, 1343-1357 (2015).

10. Normann, C. \& Buttenschon, H. N. Gene-environment interactions between HPA-axis genes and stressful life events in depression: a systematic review. Acta Neuropsychiatr. 31, 186-192 (2019).

11. Plotsky, P. M. et al. Long-term consequences of neonatal rearing on central corticotropin-releasing factor systems in adult male rat offspring. Neuropsychopharmacology 30, 2192-2204 (2005).

12. Binder, E. B. The role of FKBP5, a co-chaperone of the glucocorticoid receptor in the pathogenesis and therapy of affective and anxiety disorders. Psychoneuroendocrinology 34 (Suppl 1), S186-S195 (2009).

13. Klaassens, E. R.. Giltay, E. J., Cuijpers, P., van Veen, T. \& Zitman, F. G. Adulthood trauma and HPA-axis functioning in healthy subjects and PTSD patients: a meta-analysis. Psychoneuroendocrinology 37, 317-331 (2012).

14. Speer, K. E., Semple, S., Naumovski, N., D'Cunha, N. M. \& McKune, A. J. HPA axis function and diurnal cortisol in post-traumatic stress disorder: a systematic review. Neurobiol. Stress 11, 100180 (2019).
15. Golub, Y., Mauch, C. P., Dahlhoff, M. \& Wotjak, C. T. Consequences of extinction training on associative and non-associative fear in a mouse model of posttraumatic stress disorder (PTSD). Behav. Brain Res. 205, 544-549 (2009).

16. Golub, Y. et al. Reduced hippocampus volume in the mouse model of posttraumatic stress disorder. J. Psychiatr. Res. 45, 650-659 (2011).

17. Golub, Y. et al. Effects of In utero environment and maternal behavior on neuroendocrine and behavioral alterations in a mouse model of prenatal trauma. Dev. Neurobiol. 76, 1254-1265 (2016).

18. Bremner, J. D. Traumatic stress: effects on the brain. Dialogues Clin. Neurosci. 8 , 445-461 (2006)

19. Shin, L. M., Rauch, S. L. \& Pitman, R. K. Amygdala, medial prefrontal cortex, and hippocampal function in PTSD. Ann. N. Y. Acad. Sci. 1071 67-79 (2006).

20. Kilkenny, C., Browne, W., Cuthill, I. C., Emerson, M. \& Altman, D. G. Animal research: reporting in vivo experiments: the ARRIVE guidelines. Br. J. Pharmacol. 160, 1577-1579 (2010).

21. Seibenhener, M. L. \& Wooten, M. W. Isolation and culture of hippocampal neurons from prenatal mice. J. Vis. Exp. 65, e3634 (2012) https:/doi.org/ $10.3791 / 3634$

22. Bustin, S. A. et al. The MIQE guidelines: minimum information for publication of quantitative real-time PCR experiments. Clin. Chem. 55, 611-622 (2009).

23. Cottrell, E. C. \& Seckl, J. R. Prenatal stress, glucocorticoids and the programming of adult disease. Front. Behav. Neurosci. 3, 19 (2009).

24. Labermeier, C. et al. A polymorphism in the Crhr1 gene determines stress vulnerability in male mice. Endocrinology 155, 2500-2510 (2014).

25. Matosin, N., Halldorsdottir, T. \& Binder, E. B. Understanding the molecular mechanisms underpinning gene by environment interactions in psychiatric disorders: the FKBP5 model. Biol. Psychiatry 83, 821-830 (2018).

26. Zannas, A. S., Wiechmann, T., Gassen, N. C. \& Binder, E. B. Gene-stressepigenetic regulation of FKBP5: clinical and translational implications. Neuropsychopharmacology 41, 261-274 (2016).

27. Szymanska, M. et al. The effect of antidepressant drugs on the HPA axis activity, glucocorticoid receptor level and FKBP51 concentration in prenatally stressed rats. Psychoneuroendocrinology 34, 822-832 (2009).

28. Jankord, R. \& Herman, J. P. Limbic regulation of hypothalamo-pituitaryadrenocortical function during acute and chronic stress. Ann. N. Y. Acad. Sci. 1148, 64-73 (2008).

29. Wei, Q. et al. Glucocorticoid receptor overexpression in forebrain: a mouse model of increased emotional lability. Proc. Natl Acad. Sci. USA 101, 11851-11856 (2004)

30. Wei, Q. et al. Overexpressing the glucocorticoid receptor in forebrain causes an aging-like neuroendocrine phenotype and mild cognitive dysfunction. J. Neurosci. 27, 8836-8844 (2007).

31. Shipton, O. A. et al. Left-right dissociation of hippocampal memory processes in mice. Proc. Natl Acad. Sci. USA 111, 15238-15243 (2014).

32. Aken, B. L. et al. The Ensembl gene annotation system. Database, 2016, baw093 (2016) https://doi.org/10.1093/database/baw093.

33. McGowan, P. O. et al. Epigenetic regulation of the glucocorticoid receptor in human brain associates with childhood abuse. Nat. Neurosci. 12,342-348 (2009).

34. Weaver, I. C. et al. Epigenetic programming by maternal behavior. Nat. Neurosci. 7, 847-854 (2004).

35. Suderman, M. et al. Conserved epigenetic sensitivity to early life experience in the rat and human hippocampus. Proc. Natl Acad. Sci. USA 109 (Suppl 2), 17266-17272 (2012).

36. Müller, M. B. et al. Limbic corticotropin-releasing hormone receptor 1 mediates anxiety-related behavior and hormonal adaptation to stress. Nat. Neurosci. $\mathbf{6}$, 1100-1107 (2003). 